\title{
Valoración del envejecimiento a partir de la conectividad funcional cerebral estimada mediante medidas de sincronización electroencefalográficas
}

\author{
Julián J. González y Almudena González \\ Universidad de La Laguna (España)
}

En este trabajo estudiamos el envejecimiento cerebral fisiológico a partir del análisis de la conectividad electroencefalográfica (EEG) funcional entre distintas zonas corticales en dos grupos de sujetos sanos entre 50-65 años y entre 66-80 años. Para ello, se realizaron (en reposo y ojos cerrados) registros EEG digitales monopolares $(256 \mathrm{~Hz}$ de frecuencia de muestreo) referenciados al promedio de 16 canales/electrodos (6-frontales, 4-temporales, 2-centrales, 2-parietales, 2occipitales). Estimamos la conectividad entre canales mediante la función de coherencia en las bandas de frecuencia delta/theta/alfa/beta, y mediante un índice (L) de sincronización nolineal generalizada. Analizamos la conectividad individual entre todos los pares posibles de canales y la conectividad global de cada canal con el resto. Los resultados muestran que determinadas interdependencias individuales medidas por el índice L y por la coherencia en banda-delta, decrecen con la edad, especialmente para las conexiones centro-temporales-occipitales. Además, se observa un decrecimiento lineal significativo con la edad de los índices de interdependencia señalados, especialmente para las parejas y promedios individuales de las zonas señaladas. También las interdependencias globales, especialmente las de tipo nolineal, de ciertas zonas centro-laterales y posteriores declinan con la edad. Concluimos que, medidas individuales y globales de conectividad funcional EEG entre determinadas áreas corticales pueden ayudar a valorar objetivamente el envejecimiento neurofisiológico.

Palabras clave: Envejecimiento, conectividad funcional, electroencefalograma, coherencia, sincronización nolineal.

Assessment of aging by cortical functional connectivity estimated through synchronization electroencephalographic measurements. This work was aimed to study the physiological cerebral aging from the analysis of functional electroencephalographic (EEG) connectivity between different cortical areas in two groups of healthy subjects between $50-65$ years and between $66-80$ years. With this purpose, digital monopolar EEG recordings were carried out at rest and closed eyes, taking as sampling rate, $256 \mathrm{~Hz}$ and as reference, the average of the 16 channels recorded (6-frontals, 4temporals, 2-centrals, 2-parietal, 2-occipital). The functional cortical connectivity was estimated from the interdependence between channels using the coherence function in the frequency bands delta/theta/alpha/beta, and an index (L) of nonlinear generalized synchronization. The pairwise connectivity between all possible channels and a global connectivity between each channel and all others was computed. The results show that certain local interdependencies measured by the index L and by the delta band coherence, decreased with age; these were especially significant for the temporal-occipital-central connections. Moreover, a significant linear decrease with age of the interdependence indices aforementioned for the pair and local averages was observed. Also global interdependencies, especially those of nonlinear type, for certain central-lateral and posterior areas, decline with age. It is concludes that individual and global measures of EEG functional connectivity between certain brain areas can help to objectively evaluate the neurophysiological aging.

Keywords: Aging, functional connectivity, electroencephalogram, coherence. nonlinear synchronization.

Correspondencia: Julián Jesús González. Departamento de Fisiología. Universidad de La Laguna. Carretera La Cuesta-Taco, s/n. C.P.: 38071. Tenerife (España). E-mail: jugonzal@ull.es 
Sabemos que el envejecimiento cerebral fisiológico se caracteriza por una pérdida de los contactos sinápticos y por la apoptosis neuronal; estos factores producen una disminución con la edad, del rendimiento motor, y de funciones cognitivasconductuales como la memoria episódica, la memoria de trabajo, la función ejecutiva, y la velocidad de procesamiento sensorial (Rossini, Rossi, Babiloni y Polich, 2007; Goh, 2011). No obstante, la redundancia neural y la remodelación plástica de las redes cerebrales, junto con el entrenamiento físico y mental, capacitan a los ancianos sanos el mantenimiento de su actividad cerebral y de sus capacidades intelectuales a un nivel suficiente como para desarrollar con cierta plenitud su actividad cotidiana y afectiva. Sin embargo, la edad es el principal factor de riesgo para los trastornos neurodegenerativos como la enfermedad de Alzheimer (AD) que tienen un impacto sobre la función cognitiva. En la última década se han desarrollado estudios sobre el envejecimiento fisiológico cerebral a partir de técnicas que miden la conectividad funcional entre distintas zonas del cerebro a partir de datos de imágenes de resonancia magnética (fRMI): Sin embargo son muy pocos los realizados partir de señales EEG o MEG.

\section{Estudio de conectividad funcional con técnicas de fRMI}

Aunque los cambios neurocognitivos relacionados con la edad involucran múltiples mecanismos, una idea general extraída a partir de varios estudios de fRMI es que en los adultos jóvenes las regiones del cerebro que muestran respuestas especializadas para procesos cognitivos específicos tienden a ser menos especializadas; y responder de manera parecida a través de un gama más amplia de diferentes condiciones cognitivas en los adultos mayores. Este proceso es denominado desdiferenciación de la actividad funcional del cerebro relacionada con la edad (Park, Polk, Mikels, Taylor y Marshuetz, 2001; Li y Sikström, 2002) y puede estar relacionado con alteraciones con la edad en la conectividad entre las redes neuronales cerebrales. En esta línea se ha establecido que el envejecimiento de los mayores produce una reducción de la conectividad funcional (fRMI) entre diferentes regiones del cerebro, lo que sugiere que la comunicación entre las diferentes regiones del cerebro puede ser menos efectiva (Andrews-Hanna et al., 2007; Buckner et al., 2009; Grady et al., 2010). De hecho, estos trabajos han demostrado una reducción con la edad del carácter distintivo de las representaciones en las regiones posteriores, y un mayor compromiso frontal, lo que podría estar asociado con la comunicación funcional menos eficaz entre estas redes neuronales en los mayores. Por tanto, a fin de que el procesamiento cognitivo sea eficiente, el procesamiento dentro de una región neuronal debe operar con efectividad, y las diferentes regiones neuronales implicadas deben poder comunicarse entre sí. Como consecuencia, la actividad funcional entre estas diferentes regiones debe covariar o estar conectada de alguna manera. Se han empleado varios métodos en distintos estudios que demuestran que tal covariación funcional entre diferentes regiones corticales -que 
denominamos conectividad funcional- es un marcador importante de la capacidad cognitiva y el rendimiento (Bullmore y Sporns, 2009). En estado de reposo se ha analizado la conectividad funcional a partir de la correlación de la evolución temporal funcional de señales de baja frecuencia entre regiones cerebrales de interés predefinidas (Andrews-Hanna et al., 2007; Buckner et al., 2009). Estos estudios confirman que el envejecimiento pone en peligro la conectividad funcional entre regiones con redes por defecto o predeterminadas y otras áreas del cerebro; y corroboran los hallazgos de un gradiente posterior-anterior de descensos en la conectividad con el envejecimiento, estando más afectada las regiones anteriores.

\section{Estudios de conectividad mediante señales EEG}

En este contexto, las medidas de interdependencia que se han desarrollado para estimar la conectividad funcional entre distintas áreas/canales/electrodos del córtex han sido varias; la más antigua es la de coherencia espectral que es una medida lineal de sincronización en amplitud y fase en una determinada banda de frecuencia EEG. Recientemente, se han desarrollado medidas de interdependencia no lineal procedente de la teoría del caos determinista que dan idea de la sincronización generalizada entre dos zonas corticales y medidas de sincronización de fase -sincronización temporal- en las bandas de frecuencia del EEG (Pereda y González, 2008; González et al., 2011). Dado que en el presente trabajo se pretende aplicar estas medidas al estudio de marcadores del envejecimiento funcional cerebral, dedicaremos en el apartado de Métodos una breve descripción de estos procedimientos. Nuestro grupo de investigación ha aplicado estás técnicas al estudio de la conectividad funcional EEG en distintas patologías como el TDAH, en estudios del sueño, maduración de neonatos, etc. en humanos y en animales en diversas situaciones experimentales con resultados de interés (De la Cruz et al., 2007; Genet et al., 2012; Rial, González, Gené y Mourad, 2013; González et al., 2013). Para analizar la dirección de las interacciones entre redes corticales se han utilizado medidas de interdependencia capaces de estimar relaciones asimétricas en la conectividad funcional. Una medida utilizada para este propósito es una variante de la función de coherencia denominada coherencia parcial direccional (Schelter et al., 2006) y representa una medida de causalidad que puede proporcionar tanto la dirección como la fuerza de la interacción entre múltiples variables acopladas. En un reciente estudio con esta metodología aplicada a sujetos de distintas edades (Zhu et al., 2011) encontraron que la asimetría de las redes interactivas corticales declina con el envejecimiento produciéndose una mayor pérdida de conectividad en la zona frontal izquierdo y en las áreas centrales.

Teniendo en cuenta estas consideraciones y dado que por otro lado, la clínica del EEG es más accesible y de menor coste que la de la fRMI; el presente trabajo tiene como objetivo el análisis de marcadores del envejecimiento cerebral fisiológico a partir 
distintas medidas de la conectividad funcional EEG. Para ello se calcularan a partir de registros digitales de EEG multicanales, las matrices de sincronización lineal y no lineal del conjunto de pares de canales EEG, en una población de sujetos sanos entre 50 y 80 años de la Isla de Tenerife en vigilia/reposo en la situación de ojos cerrados. Finalmente mediante los procedimientos indicados se discutirán aquellas conectividades y más sensibles al envejecimiento cerebral fisiológico.

\section{MÉTODO}

\section{Sujetos}

El trabajo fue realizado en dos grupos de adultos sanos voluntarios: un grupo (A) de 7 sujetos con edades entre 50-65 años y un segundo grupo (B) de otros 7 sujetos con edades entre 66-80 años. Todos los sujetos seleccionados presentaban un EEG normal según informe del Servicio de Neurofisiología Clínica del Hospital Universitario La Candelaria de Tenerife.

\section{Procedimientos e instrumentos}

Se realizaron registros de EEG digitales en vigilia y reposo con los sujetos manteniendo los ojos cerrados. Los registros EEG fueron monopolares, referenciados al promedio del conjunto de 16 canales/electrodos (Fp1-2, F3-5, F7-8, T3-4, T5-6, C3-4, P3-P4, O1-O2) y siguiendo el sistema EEG estándar 10-20. Las señales EEG se muestrearon a $256 \mathrm{~Hz}$ con filtros en la banda $0,05-40 \mathrm{~Hz}$ y filtro de red alrededor de 50 Hz. Se controló la impedancia de los electrodos en un rango 3-5 kOhm. El electrooculograma, ECG y los movimientos de respiración abdominal se registraron para detectar artefactos. Para cada sujeto y para cada canal EEG se seleccionaron entre 20-30 segmentos EEG de 5 segundos (1280 muestras). Primero se seleccionaron un conjunto entre 30-50 segmentos EEG libres de artefactos; luego utilizamos un método basado en la estacionariedad de los segmentos EEG para la selección final.

\section{Métodos de análisis de la conectividad funcional EEG}

Interdependencia lineal: para el conjunto de pares de canales EEG, esta se obtiene a partir de la magnitud al cuadrado de la coherencia que representa una medida (entre 0-1) de la correlación lineal, tanto en amplitud y fase, entre dos señales a una frecuencia dada. Se computa partir de la función (compleja) de coherencia entre dos señales (x e y) a partir del espectro-cruzado entre las dos señales Pxy(f) y los espectro de potencia respectivos, $\operatorname{Pxx}(f)$ y $\operatorname{Pyy}(f)$ donde $f$ es la frecuencia. La MSC es el modulo al cuadrado de la coherencia Cxy(f).La MSC se calcula en las 4 bandas de frecuencia más utilizadas en clínica EEG: delta $0.5-4 \mathrm{~Hz}$, theta $4-8 \mathrm{~Hz}$, alfa $8-13 \mathrm{~Hz}$ y beta $13-40 \mathrm{~Hz}$. 


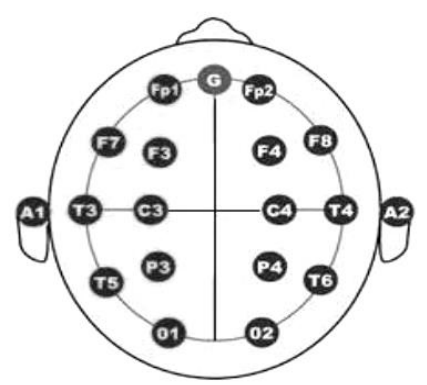

Fig. 1 (a)

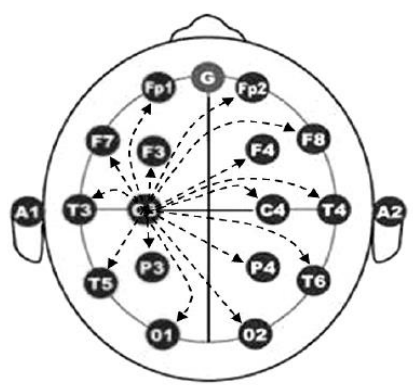

Fig. 1 (b)

Interdependencia no lineal: es una medida de la interdependencia no lineal entre dos señales (índice L) procedente de la teoría de los sistemas dinámicos no lineales. Proporciona una estimación robusta del grado de sincronización generalizada entre dos (sub) sistemas a partir de las señales que ellos generan. Con el fin de calcular $\mathrm{L}$, se construyen primero los vectores de estado retrasados Xi e Yi a partir de las señales $\mathrm{X}$ e Y: es necesario para ello establecer previamente los parámetros del embedding, esto es, del espacio donde se va a reconstruir el sistema/señal (dimensión del espacio de reconstrucción $\mathrm{m}$, retraso tau y espaciado intervectores $\mathrm{w}$ ). El índice de interdependencia $\mathrm{L}(\mathrm{X} \backslash \mathrm{Y})$ se calcula teniendo en cuenta a) los índices temporales de los $\mathrm{k}$ vecinos más próximos de $\mathrm{Xi}$ (y respectivamente de $\mathrm{Yi}$ ), b) para cada $\mathrm{Xi}$, el rango que la distancia entre $\mathrm{Xi}$ y $\mathrm{Xj}$ toma en una lista ordenada ascendente de las distancias entre $\mathrm{Xi}$ y todos los $\mathrm{Xi} \neq \mathrm{j}$, y c) el rango medio condicionado o mediado por $\mathrm{Y}$. De forma análoga se calcula $\mathrm{L}(\mathrm{Y} \backslash \mathrm{X})$ simplemente intercambiando la función $\mathrm{X}$ por $\mathrm{Y}$ en los cálculo correspondientes. Finalmente, el índice $\mathrm{L}$ de interdependencia efectivo a partir del promedio entre $\mathrm{L}(\mathrm{X} \backslash \mathrm{Y})$ y $\mathrm{L}(\mathrm{Y} \backslash \mathrm{X})$. Los valores de $\mathrm{L}$ oscilan entre 0 (nula interdependencia) y 1 (fuerte interdependencia).

Se calculó la MSC y el índice L para el conjunto de los 120 parejas posibles del conjunto de canales EEG y la MSC e índice L global de cada canal mediante el promedio del conjunto de las 15 interdependencias de cada canal con el resto de los canales. En la figura 1(a) se muestra el diagrama de los 16 canales registrados y debajo en la figura 1(b) el conjunto de las 15 parejas para uno de los canales (el ejemplo corresponde la canal centra $\mathrm{C} 3$ ).

Prueba de datos subrogados: A fin de evitar acoplamientos por conducción de volumen (ruido del conjunto de redes neuronales del cerebro que pueden afectar al conjunto de los canales EEG y producir correlaciones ficticias), la fiabilidad de los índices de interdependencia entre dos señales (X e Y) se comprueba mediante la repetición de su cálculo después de subrogar una de las señales (p.e. Y) con el fin de eliminar cualquier posible interdependencia al azar entre ellos. Las señales subrogadas se 
obtienen de manera diferente según el tipo de interdependencia a chequear entre los pares de señales. (González et al., 2013). Una vez que se obtiene las señales subrogadas (s) de Y, se calcula el valor del índice correspondiente entre la X original y las versiones subrogadas de Y, de este modo se estima numéricamente la distribución del índice bajo la hipótesis nula de independencia. La falta de significación en la interdependencia entre $\mathrm{X}$ e Y, según esta prueba, se tiene en cuenta asociando al correspondiente índice (MSC o L) entre $\mathrm{X}$ e $\mathrm{Y}$ un valor igual a cero en los cálculos.

\section{Medidas estadísticas}

Las diferencias entre grupos se valoraron aplicando la prueba U de MannWhitney de medidas independientes al conjunto de las parejas o a los promedios globales de cada canal o zona cortical considerada. Además se graficó la dispersión de los valores de MSC y L frente a la edad del conjunto de los sujetos, determinándose el coeficiente de correlación y el valor de su significación estadística.

Las medidas de interdependencia y la prueba de datos subrogados fueron calculadas a través de un software en Matlab desarrollada en nuestro laboratorio. Para las pruebas estadísticas se utilizó el programa Statistica.

\section{RESULTADOS}

\section{Interdependencia lineal}

Para la coherencia en banda delta, los pares o acoplamientos entre canales que exhibieron diferencias significativas $(p<0.01)$ con los grupos de edad fueron los siguientes: C3-T5, C3-C4 y C3-T4. Para el caso de la coherencia en banda theta estos fueron $\mathrm{C} 3-\mathrm{C} 4$, T4-O1 y $\mathrm{C} 4-\mathrm{O} 1$ con $p<0.01$. Ni la coherencia en banda alfa ni la correspondiente a la banda beta mostraron diferencia con la edad en ninguno de los 120 acoplamientos entre canales analizados.

Como ejemplos mostramos en la figura 2 izquierda el diagrama de dispersión correspondiente a la interdependencia en banda delta para el acoplamiento C3-C4. Puede observarse el decrecimiento significativo de la coherencia en banda delta con la edad con una correlación importante. El efecto de cambio con la edad también puede mostrase a partir de los contrastes entre los dos grupos de edad A y B realizados con la U de MannWhitney.

En la figura 2 derecha mostramos un ejemplo para el acoplamiento entre C3T5 en la que observamos que la coherencia delta es claramente distinta en ambos grupos: mucho menor para el grupo B de mayor edad. 

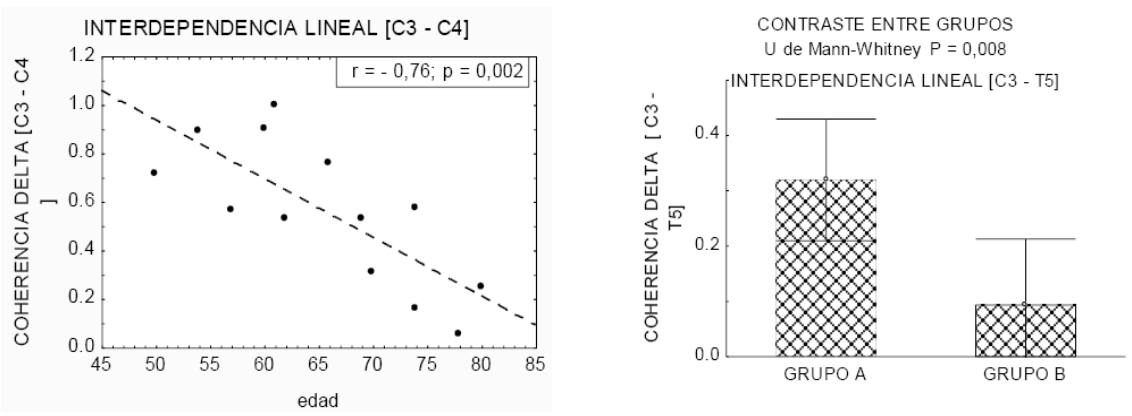

Fig. 2. Izquierda: Diagrama de dispersión para la interdependencia lineal entre C3-C4 medida por la coherencia en la banda de frecuencia delta. En la figura se muestra el valor del coeficiente de correlación $r$ entre la coherencia y la edad de los sujetos y el de la significación estadística del mismo $(p)$. Derecha: Diferencias estadísticas entre los grupos de edades A y B para las medias de la interdependencia lineal (coherencia delta) realizada a través del test $\mathrm{U}$ indicado y correspondiente al acoplamiento C3-T5.

\section{Interdependencia nolineal}

En la tabla 1 se muestra el conjunto de pares o acoplamientos entre canales que mostraron diferencias significativas siendo el grupo de menor edad B el que exhibe una menor sincronización entre los acoplamientos mostrados (ver figuras representativas). Puede observarse que el número de acoplamientos que muestran dependencia con la edad de los sujetos es muy superior que el caso de la interdependencia lineal.

En la figura 3 izquierda se muestra el diagrama de dispersión para el acoplamiento Fp1-C3 en la que se observa claramente el decrecimiento lineal con la edad para la interdependencia no lineal. En la figura 3 derecha se ha dibujado las diferencia entre las medias para los grupos A y B en relación con el índice L; se observa cómo el grupo de mayor edad (B) exhibe una menor interdependencia.

Tabla 1. Pares de canales EEG o acoplamientos que presentaban diferencias significativas entre los grupos de edades A y B para el índice L de interdependencia o sincronización nolineal

\begin{tabular}{lccc}
\hline Acoplamiento & valor de $p$ & Acoplamiento & valor de $p$ \\
\hline Fp1-C3 & $P<0.01$ & T4-T6 & $P<0.01$ \\
\hline Fp1-Fp2 & $P<0.01$ & F7-O2 & $P<0.05$ \\
\hline F7-O1 & $P<0.01$ & C3-P3 & $P<0.05$ \\
\hline C3-T4 & $P<0.01$ & C3-C4 & $P<0.05$ \\
\hline T3-P3 & $P<0.01$ & C4-O1 & $P<0.05$ \\
\hline T3-T4 & $P<0.01$ & O1-O2 & $P<0.05$ \\
\hline P3-O1 & $P<0.01$ & Fp2-C4 & $P<0.05$ \\
\hline O1-T4 & $P<0.01$ & C4-T6 & $P<0.05$ \\
\hline
\end{tabular}


En todos los casos en los que hubo diferencias significativas el valor de L del grupo (A) fue mayor que el del grupo (B).
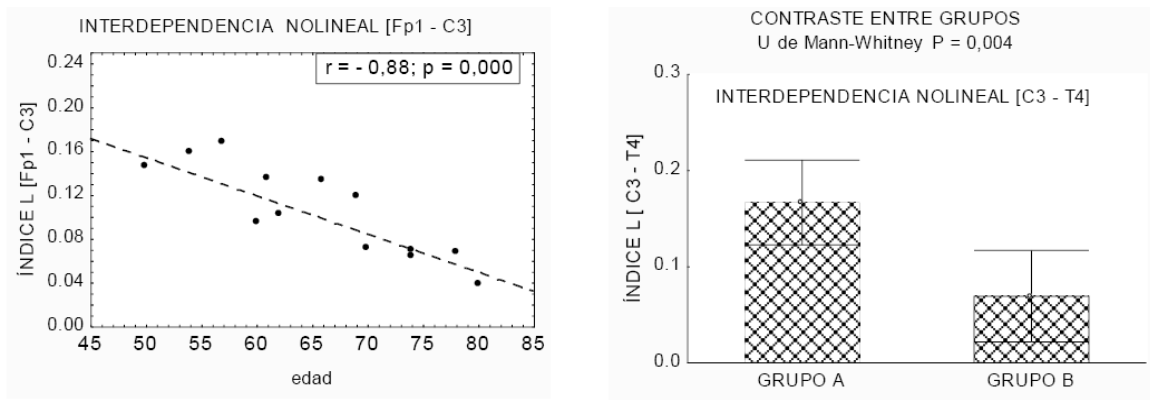

Fig. 3. Izquierda: Diagrama de dispersión para la interdependencia entre Fp1-C3 medida a través del índice L de sincronización nolineal. En la figura se muestra el valor del coeficiente de correlación ( $r$ ) entre el índice L y la edad de los sujetos y el valor $p$ de la significación estadística de $r$. Derecha: Diferencias estadísticas entre los grupos de edades A y B para las medias de la interdependencia nolineal (índice L) realizada a través del test U indicado y correspondiente al acoplamiento C3-T4.

\section{Interdependencias globales}

Nos referimos aquí a la interdependencia de cada canal EEG con el resto de los canales. Únicamente la coherencia en la banda de frecuencia delta y la interdependencia nolineal (índice L) presentaron diferencias significativas para algunos de los canales, con menor interdependencia en todos los casos para el grupo de mayor edad.

En la figura 4 hemos dibujado un mapa topográfico de los valores de $\mathrm{p}$ obtenidos de los 16 contrastes entre los grupos A y B para los valores de la interdependencia global. A la izquierda los resultados corresponden a las medidas globales de coherencia en banda delta y a la derecha los correspondientes a las medidas del índice L.

Para el caso de la coherencia delta únicamente el área centro lateral izquierda (C3, T3, F7) presenta dependencia con la edad. Mejores resultados presenta las interdependencias globales del índice L. En efecto, observamos que las regiones centroparietal-occipital izquierda (C3, P3, O1) y la centro-temporal-occipital derecha (C4, T4, O2) exhiben una alta sensibilidad en los contrastes con la edad. 

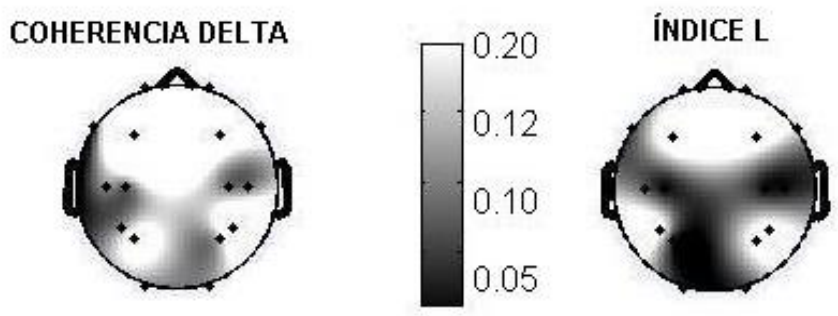

Fig. 4. Mapa topográfico de los valores de la significación estadística (Valores de P) obtenidos de los contrastes entre los valores medios de la interdependencia global de cada canal de ambos grupos de sujetos A y B. A la izquierda se muestran los resultados para la coherencia en banda delta y a la derecha los valores correspondiente al índice L. La barra del medio indica la escala de grises de valores de P. Las zonas más negras son las áreas corticales que mayor contraste presentan con la edad.

\section{DISCUSIÓN Y CONCLUSIONES}

El objetivo del trabajo era la búsqueda de marcadores del envejecimiento cerebral fisiológico a partir de medidas de conectividad funcional electroencefalográfica. Para ello, hemos utilizado técnicas digitales modernas de medidas de sincronización o interdependencia entre 16 canales EEG situados en distintas zonas de la corteza cerebral. Hemos analizado los cambios con la edad en la interdependencia entre los 120 pares o acoplamientos individuales posibles entre los distintos canales EEG y de la interdependencia global de cada canal con el resto. En el primer estudio encontramos que la conectividad funcional EEG medida por la coherencia solo presentó alteraciones con la edad para la coherencia delta y theta en unos pocos acoplamientos inter e intra hemisféricos (C3-C4, C3-T5, y C3-T4, T4-O1 y C4-O1). En relación con la coherencia entre regiones corticales se ha relatado que la edad no tiene efecto sobre la coherencia interhemisférica (Koyama, Hirasawa, Okubo y Karasawa, 1997) en contraposición con los resultados hallados por nosotros. Además estos autores relatan un decrecimiento con la edad de la coherencia intrahemisférica en todas las bandas de frecuencia del EEG. Este resultado coincide en parte con los nuestros aunque nosotros sólo hallamos un resultado similar en la coherencia delta y únicamente para el acoplamiento C3-T5. En un trabajo reciente (Zhu et al., 2011) en el que se utilizan medidas de coherencia direccional se describe una mayor pérdida de conectividad con el envejecimiento en la zona frontal izquierda y en las áreas centrales. Por el contrario, nosotros en las medidas de coherencia no encontramos cambios con la edad en los acoplamientos frontales pero si en los centrales en coincidencia con el trabajo citado. 
Si comparamos por otro lado, los resultados de los trabajos reseñados anteriormente con los encontrados por nosotros para la conectividad estimada por la interdependencia nolineal, las discrepancias y coincidencias son similares a las relatadas para el caso de la coherencia. En efecto, nosotros observamos (ver tabla 1) que la conectividad (nolineal) decrece con la edad en un mayor número de acoplamientos tanto intra como inter hemisféricos de distintas áreas corticales. Este resultado no lo podemos contrastar con otros de la literatura pues no hemos encontrado medidas de conectividad funcional EEG a través de la interdependencia nolineal en distintos rangos de edad.

También es interesante consignar que las alteraciones de la conectividad durante el envejecimiento al menos dentro del rango de edades considerado en este trabajo es de un decrecimiento de tipo lineal fundamentalmente para los acoplamientos hemisféricos mostrados C3-C4 y Fp1-C3.

Por último y considerando los resultados de la conectividad global de cada área cortical con el resto, observamos que el decrecimiento con la edad es valorable a través de la coherencia en la banda de frecuencia delta para la zona centro-lateral. Pero observamos que la conectividad estimada por la interdependencia nolineal tiene una mayor sensibilidad a los cambios con la edad, fundamentalmente la correspondiente a zonas centro-laterales y posteriores de ambos hemisferios. De nuevo no podemos contrastar estos resultados con los de otros autores al no existir trabajos similares al nuestro en la literatura.

Concluimos que el decrecimiento de la conectividad funcional cerebral durante el envejecimiento primeramente pronosticado por estudios de fRMI queda corroborado a través de los estudios de conectividad funcional cerebral a través de medidas de sincronización o interdependencia de señales EEG llevados a cabo en el presente trabajo. Hemos encontrado que determinadas conectividades funcionales electroencefalográficas de ciertas áreas corticales son claramente sensibles al envejecimiento y pueden ser utilizadas como marcadores del mismo a través de estudios de EEG en los Servicios Hospitalarios de Neurofisiología Clínica.

\section{Agradecimientos}

Este trabajo ha sido realizado con el soporte económico del Proyecto de investigación TEC2012-38453-C04-03.

\section{REFERENCIAS}

Andrews-Hanna, J.R., Snyder, A.Z, Vincent, J.L, Lustig, C., Head, D., Raic,hle M.E. y Buckner, R.L. (2007). Disruption of large-scale brain systems in advanced aging. Neuron, 56(5), 924-935.

Buckner, R.L., Sepulcre, J., Talukdar, T., Krienen, F.M., Liu, H., Hedden, T., Andrews-Hanna, J.R., Sperling, R.A. y Johnson, K.A. (2009). Cortical hubs revealed by intrinsic 
functional connectivity: mapping, assessment of stability, and relation to Alzheimer's disease. Journal of Neuroscience, 29(6), 1860-1873.

Bullmore, E. y Sporns, O. (2009). Complex brain networks: graph theoretical analysis of structural and functional systems. Nature Reviews Neuroscience, 10(3), 186-198.

De la Cruz, D.M., Mañas, S., Pereda, E., Garrido, J.M., López, S., De Vera, L. y González, J.J. (2007). Maturational changes in the interdependencies between cortical brain areas of neonates during sleep. Cerebral Córtex, 17(3), 583-90.

Genet, L., Esteban, S., González, J.J., Gamundí, A., Mourad, A. y Rial, R.V. (2012). Asymmetric sleep in rats. Laterality: Asymmetries of Body, Brain and Cognition, 17(1), 1-17.

Goh, O.S. (2011). Functional dedifferentiation and altered Connectivity in older adults: Neural Accounts of Cognitive Aging. Aging and Disease, 2(1), 30-48.

González, J.J., Mañas, S., De Vera, L., Méndez, L.D., López, S. y Garrido, J.M. (2011). Assessment of electroencephalographic functional connectivity in term and preterm neonates. Clinical Neurophysiology, 122(4), 696-702.

González, J.J., Méndez, L.D., Mañas, S., Duque, M.R., Pereda, E. y De Vera, L. (2013). Performance analysis of univariate and multivariate EEG measurements in the diagnosis of ADHD. Clinical Neurophysiology, 6(124), 1139-50.

Grady, C.L., Protzner, A.B., Kovacevic, N., Strother, S.C., Afshin-Pour, B., Wojtowicz, M., Anderson, J.A.E., Churchill, N. y McIntosh, A.R. (2010). A multivariate analysis of agerelated differences in default mode and task-positive networks across multiple cognitive domains. Cerebral Cortex, 20(6), 1432-1447.

Koyama, K., Hirasawa, H., Okubo, Y. y Karasawa, A. (1997). Quantitative EEG correlates of normal aging in the elderly. Clinical EEG (electroencephalography), 8(3), 160-165.

Li, S. y Sikström, S. (2002). Integrative neurocomputational perspectives on cognitive aging, neuromodulation, and representation. Neuroscience Biobehavioral Reviews, 26(7), 795808.

Park, D.C., Polk, T.A., Mikels, J.A., Taylor, S.F. y Marshuetz, C. (2001). Cerebral aging: integration of brain and behavioral models of cognitive function. Dialogues in Clinical Neuroscience, 3(3), 151-165.

Pereda, E. y González, J.J. (2008). Nonlinear dynamical analysis of the interdependence between central and autonomic nervous systems in neonates during sleep. Journal of Biological Physics, 3(4), 405-412.

Rial, R., González, J.J., Gené,L. y Mourad, A. (2013). Asymmetric sleep in apneic human patients. American Journal of Physiology: Regulatory, Integrative and Comparative Physiology, 304(3), 232-237.

Rossini, P.M., Rossi, S., Babiloni, C. y Polich, J. (2007). Clinical neurophysiology of aging brain: From normal aging to neurodegeneration. Progress in Neurobiology, 83(1), 375-400.

Schelter, B., Winterhalder, M., Eichler, M., Peifer, M., Hellwig, B., Guschlbauer B, Lücking, C.H., Dahlhaus, R. y Timmer, J. (2006). Testing for directed influences among neural signals using partial directed coherence. Journal of Neuroscience Methods, 152(1), 210219.

Zhu, C., Guo, X., Jin, Z., Sun, J., Qiu, Y., Zhu, Y. y Tong, S. (2011). Influences of brain development and ageing on cortical interactive networks. Clinical Neurophysiology, $122(1), 278-283$. 